\title{
AMCoR
}

Asahikawa Medical University Repository http://amcor.asahikawa-med.ac.jp/

Brain Research (2015.2) 1598:12-17.

Antinociceptive action against colonic distension by brain orexin in conscious rats.

Toshikatsu Okumuraa, Tsukasa Nozu, Shima Kumei, Kaoru Takakusaki, Saori Miyagishi, Masumi Ohhira 


\section{Antinociceptive action against colonic distension by brain orexin in conscious rats}

Toshikatsu Okumuraa , Tsukasa Nozu ${ }^{b}$, Shima Kumeia, Kaoru Takakusakic, Saori Miyagishia, Masumi Ohhira ${ }^{a}$

aDepartment of General Medicine, b Department of Regional Medicine and Education, and ${ }^{c}$ Research Center for Brain Function and Medical Engineering, Asahikawa Medical University, Midorigaoka Higashi 2-1-1-1, Asahikawa , 078-8510, Japan

Address for corresponding:

Okumura T, Department of General Medicine, Asahikawa Medical University, Midorigaoka Higashi 2-1-1-1, Asahikawa, 078-8510, JAPAN okumurat@asahikawa-med.ac.jp 


\section{Abstract}

Increasing evidence has suggested that brain orexins are implicated in a wide variety of physiological functions. With regard to gastrointestinal functions, orexin-A acts centrally to regulate gastrointestinal functions such as gastric and pancreatic secretion, and gastrointestinal motility. Visceral sensation is also known as one of key gastrointestinal functions which are controlled by the central nervous system. Little is, however, known about a role of central orexin in visceral sensation. The present study was therefore performed to clarify whether brain orexin may be involved in the process of visceral sensation. Visceral sensation was evaluated by colonic distension-induced abdominal withdrawal reflex (AWR) in conscious rats. Intracisternally administered orexin-A dose-dependently increased the threshold volume of colonic distension-induced AWR. In contrast, neither intraperitoneal injection of orexin-A nor intracisternal orexin-B altered the threshold volume. While intracisternal SB334867, an orexin 1 receptor antagonist, by itself failed to change the threshold volume, SB334867 injected centrally completely blocked the morphine-induced antinociceptive action against colonic distension. These results suggest for the first time that orexin-A specifically acts centrally in the brain to enhance antinociceptive response to colonic distension. We would furthermore suggest that endogenous orexin-A indeed mediates the antinociceptive effect of morphine on 
visceral sensation through the orexin 1 receptors. All these evidence might indicate that brain orexin play a role in the pathophysiology of functional gastrointestinal disorders such as irritable bowel syndrome because visceral hypersensitivity of the gut is considered to play a vital role in the diseases.

Key words; orexin, visceral sensation, colonic distension, antinociceptive action 


\section{Introduction}

Orexins/hypocretins are novel neuropeptides that are localized in neurons in the lateral hypothalamus (Sakurai et al., 1998; de Lecea et al., 1998). Despite their highly restricted origin, orexin nerve fibers are identified widely throughout the central nervous system (Payron et al., 1998; Date et al., 1998). These evidences suggest that activation of orexin signaling probably modulates a variety of biological systems. In fact, increasing evidence have suggested that orexins are implicated in a wide variety of physiological functions. These include feeding (Sakurai et al., 1998, Yamada et al., 2000), behavioral activity (Ida et al., 1999), sleep/awake (Chemelli et al., 1999), anxiety/depression (Suzuki et al., 2005; Brundin et al., 2007; Lutter et al., 2008; Ito et al., 2008), energy balance (Lubkin et al., 1998), neuroendocrinological response (Kuru et al., 2000) and cardiovascular functions (Shirasaka et al., 1999). In addition, orexin-A acts centrally to regulate gastrointestinal functions such as gastric and pancreatic secretion, and gastrointestinal motility (Okumura and Nozu, 2011).

Visceral sensation is also known as one of key gastrointestinal functions which are controlled by the central nervous system (Mayer and Gebhart, 1994; Longstreth et al., 2006). Little is, however, known about a role of central orexin in visceral sensation. The present study was therefore performed to clarify whether brain orexin may be involved in the 
process of visceral sensation. Visceral sensation was evaluated by colonic distension-induced abdominal withdrawal reflex in conscious rats.

\section{2. $\quad$ Results}

First, we examined the effect of exogenously administered orexin-A on visceral sensitivity. Figure 1 demonstrates the effect of intracisternal orexin-A on the threshold volume of colonic distension-induced abdominal withdrawal reflex (AWR) in rats. Intracisternally administered orexin-A dose-dependently increased the threshold volume of colonic distension-induced AWR. In contrast, intraperitoneal injection of Orexin-A at a dose of $10 \mu \mathrm{g}$ failed to alter the threshold volume (Table 1), suggesting that orexin-A acts centrally in the brain to enhance antinociceptive response to colonic distension. In addition, intracisternal injection of orexin-B at a dose of $10 \mu \mathrm{g}$ did not change the threshold volume (Table 1), suggesting that orexin-A specifically possesses an antinociceptive action to colonic distension. In the next step, we tried to clarify whether endogenous orexin-A is indeed involved in the antinociceptive action against colonic distension. As shown in Figure 2, morphine, a well-known drug for pain, significantly increased the threshold volume of colonic distension-induced AWR. While intracisternal SB334867, an OX1R antagonist, by itself failed to change the threshold volume, SB334867 injected centrally completely 
blocked the morphine-induced antinociceptive action against colonic distension, suggesting that endogenous orexin-A indeed mediates the antinociceptive effect of morphine.

\section{Discussion}

The present study demonstrated for the first time that orexin-A acts in the brain to induce an antinociceptive action against colonic distension. With regard to the role of central orexin in digestive functions, it has been reported that orexin acts in the brain to stimulate gastric and pancreatic secretion, and increase gastric and colonic motility in rats (Takahashi et al., 1999; Okumura et al., 2001; Yamada et al., 2005; Nozu et al., 2011; Nozu et al., 2012; Bülbül et al., 2010a and 2010b; Kobashi et al., 2002; Krowicki et al., 2002; Miyasaka et al., 2002). The present study therefore provided a novel evidence that central orexin plays a role in not only gastrointestinal secretion and motility but also gut sensation. To our knowledge, CRF is an only peptide in the brain that has been reported to be implicated in the colonic distension-induced AWR in rats as following. Gue et al. (1997) have reported that intracerebroventricular CRF enhanced the number of abdominal cramps evoked by rectal distension and alpha-helical CRF9-41 antagonized the CRF-induced enhancement of abdominal cramps in rats. In addition, centrally administered CRF induced a colonic hypersensitivity in Fisher 344 rats (Greewood-Van Meerveld et al., 2005). Thus, CRF acts 
centrally to increase visceral pain sensation. In contrast, orexin-A decreased the colonic distension-induced pain sensitivity as shown in this study. We would therefore suggest that orexin might be a unique peptide in the brain that is capable of inducing an antinociceptive action against colonic distension.

The present data demonstrated that an enhanced antinociceptive action against colonic distension was seen after intracisternal injection of orexin-A but not orexin-B, suggesting that orexin-A plays a role in the regulation of visceral sensation in a specific manner. According to the results by Sakurai et al. (1998), orexins binds to two specific receptors named orexin 1 receptor (OX1R) and orexin 2 receptor (OX2R). Orexin-A has high affinity for OX1R but orexin-B has significantly lower affinity for OX1R. In contrast, orexin-A and orexin-B have high affinity for OX2R equally. Based upon these results, they suggested that OX2R is a nonselective receptor for both orexin-A and $-\mathrm{B}$, while OX1R is selective for orexin-A. Considering the characterization of orexin receptors, the present results that centrally administered orexin-A but not orexin-B enhanced the AWR threshold volume may indicate that OX1R mediates the orexin-A-induced alternation of visceral sensation.

The involvement of OX1R in the enhanced AWR threshold volume was furthermore supported by the present finding that intracisternal SB334867, a specific OX1R antagonist, completely blocked the 
antinociceptive action of morphine. The above result additionally provided a solid evidence that endogenous orexin- $\mathrm{A}$ in the brain is indeed involved in the regulation of visceral sensation. Morphine induced analgesia is mediated through spinal and supraspinal mechanisms (Barton et al., 1980). In the current study, the subcutaneous administration of morphine produced an antinociceptive effect against colonic distension. The antinociceptive effects of morphine administration were blocked by a selective OX1R antagonist, SB-334867, suggesting that some parts of the analgesic effect of morphine on visceral sensation are mediated by orexinergic system through the activation of OX1R.

Orexin nerve fibers are identified widely throughout the central nervous system. Among them, regions shown to play a role in pain modulation such as periaqueductal gray (PAG) and rostral ventromedial medulla (RVM) are also innervated by orexin (Peyron et al., 1998). The lateral hypothalamus that exclusively expresses orexin has a key role in the modulation of nociceptive behavior through the PAG and RVM (Behbehani et al., 1988; Holden and Pizzi, 2008). These findings suggest that orexin may act in the PAG and/or RVM neurons to induce the antinociceptive action against colonic distension.

Irritable bowel syndrome (IBS) is a functional disorder of the gastrointestinal (GI) tract (Longstreth et al., 2006; Drossman, 2006). Brain-gut interaction plays an important role in the pathophysiology of IBS. 
Several studies have shown that visceral hypersensitivity to mechanical distension of the gut in IBS is related to symptom severity and it is considered to play a role in the pathophysiology of IBS (Mayer and Gebhart, 1994; van der Veek et al., 2008; Kanazawa et al., 2008; Kuiken et al., 2005; Posserud et al., 2007). The present findings that brain orexin plays a regulatory role in visceral sensation, suggesting that orexin in the central nervous system might be involved in the development of IBS. Based upon the above speculation, we would suggest that orexinergic signaling in the brain may be a novel therapeutic target for patients with IBS.

\section{Experimental procedures}

\subsection{Animals}

Male Sprague-Dawley rats weighing about $200 \mathrm{~g}$ were housed under controlled light/dark conditions (lights on: 07:00-19:00) with the room temperature regulated to $23-25^{\circ} \mathrm{C}$. Rats were allowed free access to standard rat chow (Solid rat chow, Oriental Yeast Co., Tokyo, Japan) and tap water. All experiments were performed in conscious animals deprived of food for $24 \mathrm{~h}$ but with free access to water up to the initiation of the experiments.

\subsection{Chemicals}


Synthetic orexin-A (human/bovine/rat/mouse) and orexin-B (rat/mouse) was purchased from Peptide Institute Inc., Osaka, Japan and were dissolved in normal saline just before the experiments. Selective orexin-A antagonist, SB-334867 (Tocris Bioscience, Ellisville, MO) (Smart et al., 2001) was dissolved in dimethyl sulfoxide (DMSO, Sigma-Aldrich, St. Louis, MO). Morphine hydrochloride (Hokuyaku Co., Hokkaido, Japan) was dissolved in saline.

4.3. Implantation of electrodes and placement of colorectal balloon Electrodes for measuring abdominal muscle contractions electrophysiologically were acutely implanted on the day of the experiment as described previously (Nozu et al., 2014). Briefly, under ether anesthesia, skin incision about $5 \mathrm{~mm}$ in length was made in rats. The electrodes (Teflon coated stainless steel, $0.05 \mathrm{~mm}$ diameter, MT Giken, Tokyo, Japan) were inserted approximately $2 \mathrm{~mm}$ into left side external oblique musculature through the incision and fixed by cyanoacrylate instant adhesive (Aron Alpha, TOAGOSEI, Tokyo, Japan) together with the incised skin. The electrode leads were externalized through this closed incision and threaded through a urethane tube. Immediately after implantation of electrorodes, a distension balloon was inserted intra-anally with the distal end positioned $2 \mathrm{~cm}$ proximal to the anus. A 6-Fr $(2 \mathrm{~mm}$ external diameter) disposable silicon balloon-urethral catheter for pediatric 
use (JU-SB0601, Terumo Co., Tokyo, Japan) was used. The maximal inflation volume for the balloon was $1.5 \mathrm{~mL}$ and the length of the maximally inflated balloon was $1.2 \mathrm{~cm}$. The balloon was secured in place by taping the catheter to the tail.

\subsection{Detection of visceral sensitivity}

Visceral pain in response to colonic distension was assessed by abdominal muscle contractions in conscious rats, which was validated as quantitative measure of visceral nociception (Ness and Gebhart, 1988). After completing the surgery for electrodes implantation and balloon placement, the rats were placed in Bollman cages while still sedated, and were allowed to recover and adjust for $20 \mathrm{~min}$ before testing. Then electrode leads were connected to a custom made electromyogram (EMG) amplifier. EMG signals were amplified, filtered $(3000 \mathrm{~Hz})$ and digitized by a PowerLab system, and stored by computer software (LabChart 7). Colonic distension was performed according to a previous publication (Tang et al., 2013). After the animals were fully awake and adjusted to the environment, ascending-limit phasic distension was applied in increments of $0.1 \mathrm{ml}(0.1-1.2 \mathrm{~mL})$ was applied for $5 \mathrm{~s}$ every $10 \mathrm{~s}$ to induce colonic distension until a visible contraction of the abdominal wall was observed by an investigator blinded to the treatment. The balloon was distended with water and the abdominal withdrawal reflex (AWR) was 
detected to examine the pain threshold volume by EMG as previously described (Qin et al., 2012). The pain threshold volume was defined as the intensity of colonic distension that elicited an observable AWR, i.e., a sudden and persistent abdominal muscle contraction.

\subsection{Experimental procedures}

We initially examined the dose-related effects of intracisternal injection of orexin-A on the colonic distension-induced AWR threshold volume. Rats received intracisternal injections of several doses of orexin-A. The doses used in this study were basically selected according to previous reports (Takahashi et al., 1999). Control animals were injected with normal saline $(10 \mu \mathrm{L})$ intracisternally. Intracisternal injection was performed under brief ether anesthesia with a $10-\mu \mathrm{L}-$ Hamilton microsyringe after rats were mounted in a stereotaxic apparatus (David Kopf Instruments, Ti- junga, CA) as reported previously (Okumura et al., 1994). Intraperitoneal injection of $10 \mu \mathrm{g}$ of orexin-A dissolved in $0.5 \mathrm{~mL}$ of saline was performed to examine the possibility that centrally administered orexin-A was acting periphery by leaking into the circulation. As a control, normal saline $(0.5 \mathrm{~mL})$ alone was injected intraperitoneally. Next, we examined the effects of intracisternal injection of orexin-B on visceral sensation. To clarify whether endogenous orexin may be involved in the regulation of visceral sensation, we examined the effect of intracisternal injection of SB334867, a specific OX1R antagonist, on the subcutaneously administered morphine-induced antinociceptive action against colonic distension. Following the intracisternal or intraperitoneal injection, and/or subcutaneous injection, rats received 
implantation of electrorodes and placement of balloon, and were moved to ballman cages to evaluate the AWR threshold volume as described above.

\subsection{Statistical analysis}

For statistical analysis of the data, data were expressed as means \pm S.E. Student's t-test and one-way ANOVA followed by Dunnett's multiple comparison test were used. Values of $\mathrm{P}<0.05$ were considered statistically significant.

\subsection{Ethical considerations}

The approval of the Research and Development and Animal Care committees at the Asahikawa Medical University was obtained for all studies.

\section{Acknowledgments}

This work was supported in part by grants-in-aid from the Ministry of Education, Science, Sports and Culture of Japan [C-26460955 (TO) and C-26460287 (TN)]. 
References

Barton, C., Basbaum, A.I., Fields, H.L., 1980. Dissociation of supraspinal and spinal actions of morphine: a quantitative evaluation. Brain Res. 188, 487-498.

Behbehani, M.M., Park, M.R., Clement, M.E., 1998. Interactions between the lateral hypothalamus and the periaqueductal gray. J. Neurosci. 8, 2780-2787.

Brundin, L., Bjorkqvist, M., Petersen, A., Träskman-Bendz. L., 2007. Reduced orexin levels in the cerebrospinal fluid of suicidal patients with major depressive disorder. Eur. Neuropsychopharmacol. 9, 573-579.

Bülbül, M., Babygirija, R., Ludwig, K., Takahashi, T., 2010a. Central orexin-A increases gastric motility in rats. Peptides 31, 2118-2122.

Bülbül, M., Babygirija, R., Zheng, J., Ludwig, K.A., Takahashi, T., 2010b. Central orexin-A changes the gastrointestinal motor pattern from interdigestive to postprandial in rats. Auton. Neurosci. 158, 24-30. 
Chemelli, R.M., Willie, J.T,, Sinton, C.M,, Elmquist, J.K,, Scammell, T., Lee, C., Richardson, J.A., Williams, S.C., Xiong, Y., Kisanuki, Y., Fitch, T.E., Nakazato, M., Hammer, R.E., Saper, C.B., Yanagisawa, M., 1999. Narcolepsy in orexin knockout mice: molecular genetics of sleep regulation. Cell 98, 437-451.

Date, Y., Ueta, Y., Yamashita, H., Yamaguchi, H., Matsukura, S., Kangawa, K., Sakurai, T., Yanagisawa, M., Nakazato, M., 1999. Orexins, orexigenic hypothalamic peptides, interact with autonomic, neuroendocrine and neuroregulatory systems. Proc. Natl. Acad. Sci. USA. 96, 748-753.

de Lecea, L., Kilduff, T.S., Peyron, C., Gao, X., Foye, P.E., Danielson, P.E., Fukuhara, C., Battenberg, E.L., Gautvik, V.T., Bartlett, F.S. 2nd, Frankel, W.N., van den Pol, A.N., Bloom, F.E., Gautvik, K.M., Sutcliffe, J.G., 1998. The hypocretins: hypothalamus-specific peptides with neuroexcitatory activity. Proc. Natl. Acad. Sci. U S A 95, 322-327.

Drossman, D.A., 2006. The functional gastrointestinal disorders and the Rome III process. Gastroenterology 130, 1377-1390.

Greenwood-Van Meerveld, B., Johnson, A.C., Cochrane, S., Schulkin, J., Myers, D.A., 2005. Corticotropin-releasing factor 1 receptor-mediated 
mechanisms inhibit colonic hypersensitivity in rats. Neurogastroenterol. Motil. 17, 415-422.

Gué, M., Del Rio-Lacheze, C., Eutamene, H., Théodorou, V., Fioramonti, J., Buéno, L., 1997. Stress-induced visceral hypersensitivity to rectal distension in rats: role of CRF and mast cells. Neurogastroenterol. Motil. 9, 271-279.

Holden, J.E., Pizzi, J.A., 2008. Lateral hypothalamic-induced antinociception may be mediated by a substance $\mathrm{P}$ connection with the rostral ventromedial medulla. Brain Res. 1214, 40-49.

Ida, T., Nakahara, K., Katayama, T., Murakami, N., Nakazato, M., 1999. Effect of lateral cerebroventricular injection of the appetite-stimulating neuropeptide, orexin and neuropeptide $\mathrm{Y}$, on the various behavioral activities of rats. Brain Res. 821, 526-529.

Ito, N., Yabe, T., Gamo, Y., Nagai, T., Oikawa, T., Yamada, H., Hanawa, T., 2008. I.c.v. administration of orexin-A induces an antideprresive-like effect through hippocampal cell proliferation. Neuroscience 157, $720-732$. 
Kanazawa, M., Palsson, O.S., Thiwan, S.I., Turner, M.J., van Tilburg, M.A., Gangarosa, L.M., Chitkara, D.K., Fukudo, S., Drossman, D.A., Whitehead, W.E., 2008. Contributions of pain sensitivity and colonic motility to IBS symptom severity and predominant bowel habits. Am. J. Gastroenterol. 103, 2550-2561.

Kobashi, M., Furudono, Y., Matsuo, R., Yamamoto, T., 2002. Central orexin facilitates gastric relaxation and contractility in rats. Neurosci. Lett. $332,171-174$.

Krowicki, Z.K., Burmeister, M.A., Berthoud, H.R., Scullion, R.T., Fuchs, K., Hornby, P.J., 2002. Orexins in rat dorsal motor nucleus of the vagus potently stimulate gastric motor function. Am. J. Physiol. Gastrointest. Liver Physiol. 283:G465-472.

Kuiken, S.D., Lindeboom, R., Tytgat, G.N., Boeckxstaens, G.E., 2005. Relationship between symptoms and hypersensitivity to rectal distension in patients with irritable bowel syndrome. Aliment. Pharmacol. Ther. 22, $157-164$.

Kuru, M., Ueta, Y., Serino, R., Nakazato, M., Yamamoto, Y., Shibuya, I., 
Yamashita, H., 2000. Centrally administered orexin/hypocretin activates HPA axis in rats. Neuroreport 11, 1977-1980.

Longstreth, G.F., Thompson, W.G., Chey, W.D., Houghton, L.A., Mearin, F., Spiller, R.C., 2006. Functional bowel disorders. Gastroenterology $1480-1491$.

Lubkin, M., Stricker-Krongrad, A., 1998. Independent feeding and metabolic actions of orexins in mice. Biochem. Biophys. Res. Commun. $253,241-245$.

Lutter, M., Krishnan, V., Russo, S.J., Jung, S., McClung, C.A., Nestler, E.J., 2008. Orexin signaling mediates the antidepressant-like effect of calorie restriction. J. Neurosci. 28, 3071-3075.

Mayer, E.A., Gebhart, G.F., 1994. Basic and clinical aspects of visceral hyperalgesia. Gastroenterology 107, 271-293.

Miyasaka, K., Masuda, M., Kanai, S., Sato, N., Kurosawa, M., Funakoshi, A., 2002. Central Orexin-A stimulates pancreatic exocrine secretion via the vagus. Pancreas $25,400-404$. 
Ness, T.J., Gebhart, G.F., 1988. Colorectal distension as a noxious visceral stimulus: physiologic and pharmacologic characterization of pseudaffective reflexes in the rat. Brain Res. 450, 153-169.

Nozu, T., Kumei, S., Takakusaki, K., Ataka, K., Fujimiya, M., Okumura, T., 2011. Central orexin-A increases colonic motility in conscious rats. Neurosci. Lett. 498, 143-146.

Nozu, T., Takakusaki, K., Okumura, T., 2014. A Balance Theory of Peripheral Corticotropin-Releasing Factor Receptor Type 1 and Type 2 Signaling to Induce Colonic Contractions and Visceral Hyperalgesia in Rats. Endocrinology 155, 4655-4664.

Nozu, T., Tuchiya, Y., Kumei, S., Takakusaki, K., Ataka, K., Fujimiya, M., Okumura, T., 2012. Endogenous orexin-A in the brain mediates 2-deoxy-D-glucose-induced stimulation of gastric motility in freely moving conscious rats. J. Gastroenterol. 47, 404-411.

Okumura, T., Fukagawa, K., Tso, P., Taylor, I.L., Pappas, T.N., 1994. Intracisternal injection of apolipoprotein A-IV inhibits gastric secretion in pylorus-ligated conscious rats. Gastroenterology 107, 1861-1864. 
Okumura, T., Nozu, T., 2011. Role of brain orexin in the pathophysiology of functional gastrointestinal disorders. J. Gastroenterol. Hepatol. 26 Suppl 3, 61-66.

Okumura, T., Takeuchi, S., Motomura, W., Yamada, H., Egashira, S., Asahi, S., Kanatani, A., Ihara, M., Kohgo, Y., 2001. Requirement of intact disulfide bonds in orexin-A-induced stimulation of gastric acid secretion that is mediated by OX1 receptor activation. Biochem. Biophys. Res. Commun. 280, 976-981.

Peyron, C., Tighe, D.K., van den Pol, A.N., de Lecea, L., Heller, H.C., Sutcliffe, J.G., Kilduff, T.S., 1998. Neurons containing hypocretin (orexin) project to multiple neuronal systems. J. Neurosci. 18, 9996-10015.

Posserud, I., Syrous, A., Lindstrom, L., Tack, J., Abrahamsson, H., Simren,

M., 2007. Altered rectal perception in irritable bowel syndrome is associated with symptom severity. Gastroenterology 133, 1113-1123.

Qin, H.Y., Xiao, H.T., Wu, J.C., Berman, B.M., Sung, J.J., Bian, Z.X., 2012. Key factors in developing the trinitrobenzene sulfonic acid-induced 
post-inflammatory irritable bowel syndrome model in rats. World J. Gastroenterol. 18, 2481-2492.

Sakurai, T., Amemiya, A., Ishii, M., Matsuzaki, I., Chemelli, R.M., Tanaka, H., Williams, S.C., Richardson, J.A., Kozlowski, G.P., Wilson, S., Arch, J.R., Buckingham, R.E., Haynes, A.C., Carr, S.A., Annan, R.S., McNulty, D.E., Liu, W.S., Terrett, J.A., Elshourbagy, N.A., Bergsma, D.J., Yanagisawa, M., 1998. Orexins and orexin receptors: a family of hypothalamic neuropeptides and $\mathrm{G}$ protein-coupled receptors that regulate feeding behavior. Cell 92, 573-585.

Shirasaka, T., Nakazato, M., Matsukura, S., Takasaki, M., Kannan, H., 1999. Sympathetic and cardiovascular actions of orexins in conscious rats. Am. J. Physiol. Regul. Integr. Comp. Physiol. 277, R1780-1785.

Smart, D., Sabido-David, C., Brough, S.J., Jewitt, F., Johns, A., Porter, R.A., Jerman, J.C., 2001. SB-334867-A: the first selective orexin-1 receptor antagonist. Br. J. Pharmacol. 132, 1179-1182.

Suzuki, M., Beuckmann, C.T., Shikata, K., Ogura, H., Sawai, T., 2005. Orexin-A (hypocretin-1) is possibly involved in generation of anxiety-like behavior. Brain Res. 1044, 116-121. 
Takahashi, N., Okumura, T., Yamada, H., Kohgo, Y., 1999. Stimulation of gastric acid secretion by centrally administered orexin-A in conscious rats. Biochem. Biophys. Res. Commun. 254, 623-627.

Tang, Q.L., Lai, M.L., Zhong, Y.F., Wang, A.M., Su, J.K., Zhang, M.Q., 2013. Antinociceptive effect of berberine on visceral hypersensitivity in rats. World J. Gastroenterol. 19, 4582-4589.

van der Veek, P.P., Van Rood, Y.R., Masclee, A.A., 2008. Symptom severity but not psychopathology predicts visceral hypersensitivity in irritable bowel syndrome. Clin. Gastroenterol. Hepatol. 6, 321-328.

Yamada, H., Okumura, T., Motomura, W., Kobayashi, Y., Kohgo, Y., 2000. Inhibition of food intake by central injection of anti-orexin antibody in fasted rats. Biochem. Biophys. Res. Commun. 267, 527-531.

Yamada, H., Takahashi, N., Tanno, S., Nagamine, M., Takakusaki, K., Okumura, T., 2005. A selective orexin-1 receptor antagonist, SB334867, blocks 2-DG-induced gastric acid secretion in rats. Neurosci. Lett. 376, $137-142$. 



\section{Figure legends}

Figure 1.

Effect of intracisternal injection of orexin-A on the colonic distension-induced abdominal withdrawal reflex (AWR) threshold volume in conscious rat. Each column represents the mean \pm S.E. Number of rats was shown in the parenthesis. ${ }^{*} \mathrm{P}<0.01$, when compared with control.

Figure 2.

Effect of intracisternal injection of SB334867, an OX1R antagonist, on the subcutaneously administered morphine-induced antinociceptive action against colonic distension in conscious rats. Each column represents the mean \pm S.E. Number of rats was shown in the parenthesis. $* \mathrm{P}<0.01$, when compared with saline. $\quad * * \mathrm{P}<0.01$, when compared with DMSO + morphine. SB; SB334867. 
Figure 1

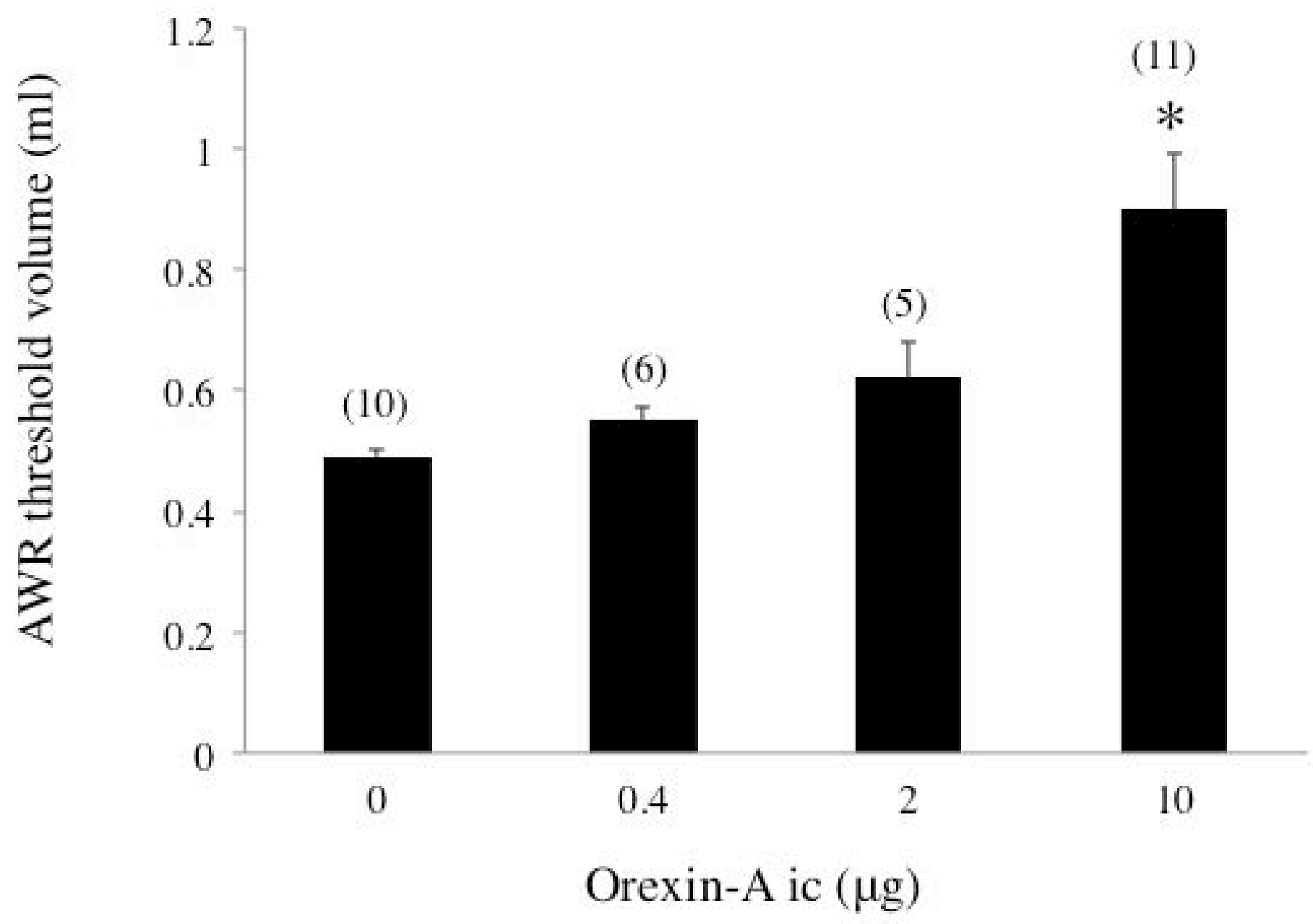


Figure 2
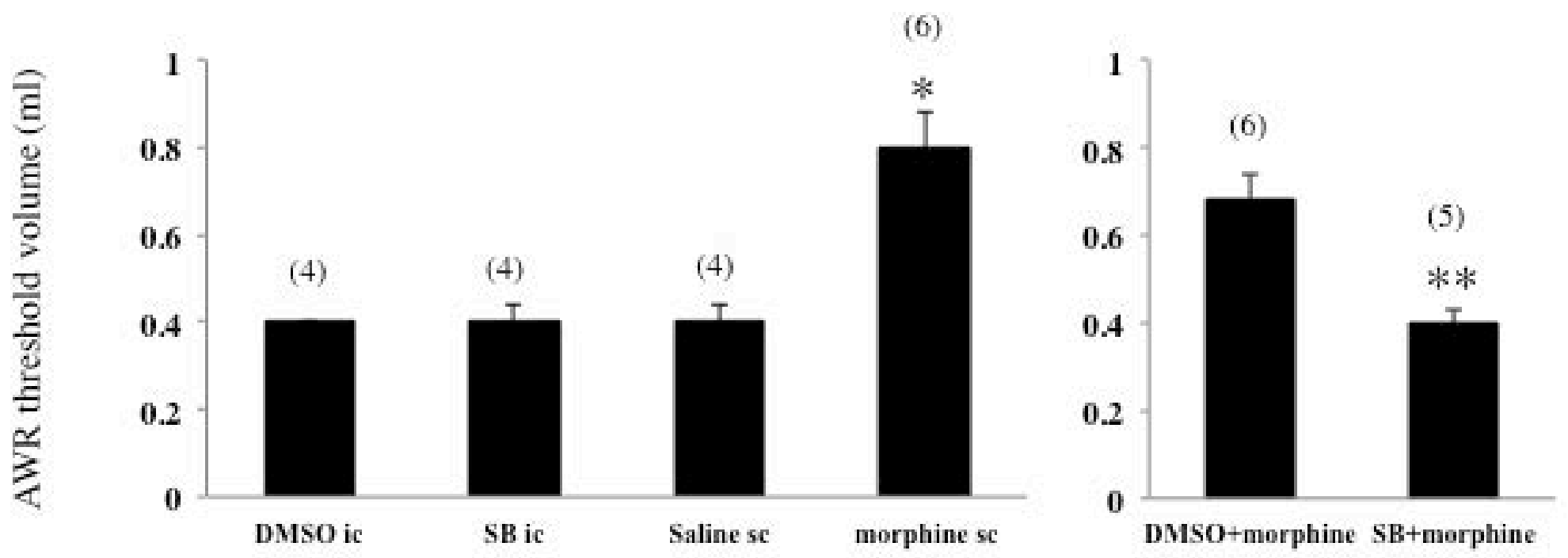
Table 1. Effect of intraperitoneal orexin-A and intracisternal orexin-B on colonic distension-induced visceral pain in rats

\begin{tabular}{llc}
\hline & $\mathrm{N}$ & AWR threshold volume (ml) \\
\hline Saline (ip) & 5 & $0.48 \pm 0.02$ \\
Orexin-A $10 \mu \mathrm{g}$ (ip) & 6 & $0.47 \pm 0.03$ \\
Saline (ic) & 4 & $0.43 \pm 0.03$ \\
Orexin-B $10 \mu \mathrm{g}$ (ic) & 5 & $0.46 \pm 0.04$ \\
\hline
\end{tabular}

Each data represents the mean \pm S.E. AWR; abdominal withdrawal reflex. ip; intraperitoneal, ic; intracisternal 